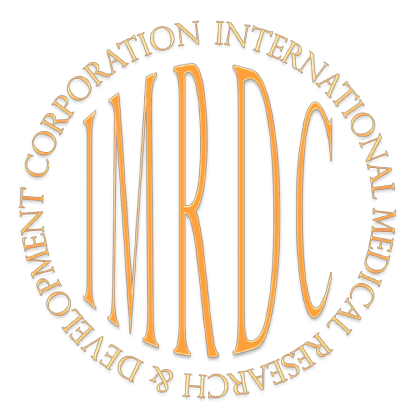

\title{
An Overview of Labor Pain: Components and Stages of the Labor Process
}

\author{
Hend A. El-Sakhawy ${ }^{1}$, Ahmed M. Abodonya ${ }^{2,3}$, Walid Kamal Abdelbasset ${ }^{4,5}$, \\ Mohamed A. El-Sakhawy ${ }^{6,7 *}$ \\ ${ }^{1}$ Department of Anesthesiology and ICU, Faculty of Medicine (Girls), Al-Azhar University, Cairo, Egypt \\ ${ }^{2}$ Department of Surgery, College of Medicine, Prince Sattam bin Abdulaziz University, Al-Kharj, \\ Saudi Arabia \\ ${ }^{3}$ Department of Anesthesia and Intensive Care, Faculty of Medicine, Al-Azhar University, Cairo, Egypt \\ ${ }^{4}$ Department of Health and Rehabilitation Sciences, College of Applied Medical Sciences, \\ Prince Sattam bin Abdulaziz University, Al-Kharj, Saudi Arabia \\ ${ }^{5}$ Department of Physical Therapy, Kasr Al-Aini Hospital, Cairo University, Giza, Egypt \\ ${ }^{6}$ Department of Medical Laboratory Sciences, College of Applied Medical Sciences, \\ Prince Sattam bin Abdulaziz University, Al-Kharj, Saudi Arabia \\ ${ }^{7}$ Department of Medicinal and Aromatic Plants, Desert Research Center, Cairo, Egypt
}

\begin{abstract}
Labor pain (LP) is unbearable and a major source of anxiety and stress. Painful uterine contractions cause hyperventilation in the mother, and because of augmented catecholamine concentration, both the mother and her fetus will be hypoxic. Effective analgesia provides protection from difficulties and ensures good results in both the mother and fetus. Hence, the control of pain should form an integral part of labor management at any level. This brief review aims to identify LP and its effects on fetus and mother, stages of delivery and labor process, and components of LP. (International Journal of Biomedicine. 2021;11(3):315-317)
\end{abstract}

Key Words: labor pain $\bullet$ fetus $\bullet$ visceral pain $\bullet$ somatic pain $\bullet$ sensory fiber

For citation: El-Sakhawy HA, Abodonya AM, Abdelbasset WK, El-Sakhawy MA. An Overview of Labor Pain: Components and Stages of the Labor Process. International Journal of Biomedicine. 2021;11(3):315-317. doi:10.21103/Article11(3)_BR

$\mathrm{L}$ abor is the complex and painful process through which a fetus and placenta are delivered from the uterus. The exact mechanisms triggering the onset of labor remain unknown. Some triggers of labor onset that have been suggested are an increase in myometrial OT receptor concentration, improved production of prostaglandin, and enlarged myometrial gap-junctions. ${ }^{(1)}$

Effects of labor pain on the fetus and mother

Pain is the most predominant signal of the beginning of labor. Labor pain is unbearable and a major source of anxiety

*Corresponding author: Dr. Mohamed A. El-Sakhawy. Department of Medical Laboratory Sciences, College of Applied Medical Sciences, Prince Sattam bin Abdulaziz University, Al-Kharj, Saudi Arabia.E-mail: msakhawy@hotmail.com and stress. Painful uterine contractions cause hyperventilation in the mother, and because of augmented catecholamine concentration, both the mother and her fetus will be hypoxic. Effective analgesia provides protection from difficulties and ensures good results in both the mother and fetus. Hence, the control of pain should form an integral part of labor management at any level. In addition, effective analgesia is crucial in some critical cases, as in women with cardiac problems and Grade II and Grade III dyspnea. ${ }^{(2,3)}$

LP by itself, aside from childbirth tissue injury, is directly or indirectly related to special influences on the mothers and fetuses. The response to the pain induces obvious stimuli to circulation and respiration, in addition to the function of neuroendocrine, especially in the hypothalamic autonomic centers and limbic structures, as well as in psychodynamic 
actions, such as apprehension and nervousness, which induce harmful consequences, which may occur to the mother and fetus. Appropriate pain relief is the only solution to get rid of and/or mitigate these responses. ${ }^{(4)}$

LP is one of the potent respiratory stimuli, causing an increase in ventilation and consumption of oxygen during contractions. Hyperventilation during contractions may cause temporary hypoxemia in the mother and, potentially, fetal hypoxemia. Respiratory depression results from analgesic procedures (eg, systemic opioid analgesia) and may exacerbate hyperventilation during labor. The hyperventilation causes acute respiratory alkalosis and leftward shifting of the mother's oxyhemoglobin degradation curve, reducing the transfer of oxygen to the fetus. Labor stress and pain also stimulate the sympathetic nervous system, leading to elevation of plasma catecholamine concentrations. Levels of norepinephrine and epinephrine are elevated during unmedicated labor $(200 \%-$ $600 \%$ ) in addition to elevation of catecholamines during reduced uterine blood flow. ${ }^{(4,5)}$

Activation of sympathetic response by pain, induces cardiac output in a potentially harmful way in patients suffering from heart disease, anemia and preeclampsia. Additionally, it reduces gastric evacuation, which may lead to vomiting and nausea, and reduces the propulsive movement of the intestine, which may cause ileus and oliguria. The pituitary and placenta produce a great amount of $\beta$-endorphin to the blood but noticeably not enough to decrease pain successfully. ${ }^{(6)}$

Pain intensity and complaints related to vaginal delivery and labor differ extensively in parturients. The pain rating index (PRI) is lower in parous mothers than nulliparous mothers. In addition, an important variance between parous and nulliparous in pain sensory quality has been detected. The scores of pain vary from mild to severe (Figure 1); the PRI in women during labor is commonly eight to ten times greater than in those patients suffering from post-herpetic neuralgia, phantom limb pain and cancer pain. ${ }^{(4,5)}$

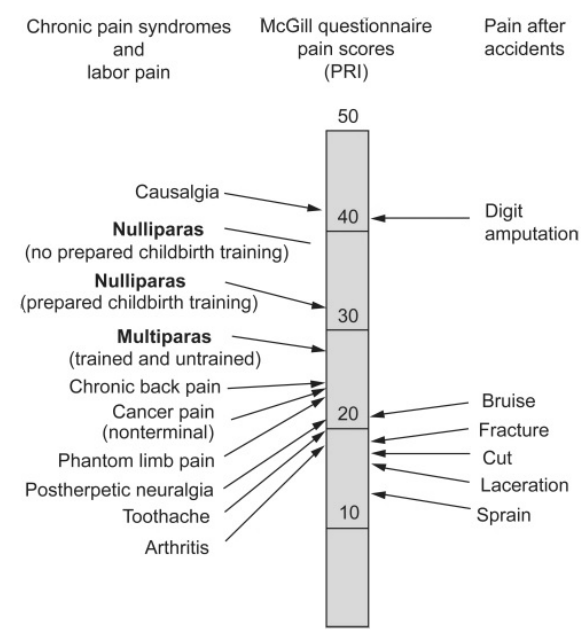

Fig. 1. Pain intensity according to the McGill Pain Questionnaire. ${ }^{(1)}$

Finally, severe mental health impairment may result from continuous severe pain, which affects the maternalneonatal connection and sexual relationships, and may lead to post-partum depressive disorder, and, rarely, to post-traumatic stress disorder. ${ }^{(4)}$

\section{Sensory Nerve Supply of the Birth Canal}

Sensory fibers from the uterus pass by sympathetic nerves through the inferior hypogastric plexus (T10-L1) and from the cervix by parasympathetic nerves (S2-4). The pudendal nerve (S2-4) supplies vaginal and pelvic outlets. Minor supplies are also derived from the perineal branch of the posterior (L2-4) and genito-femoral nerves. (L1, 2) (Figure 2). ${ }^{(7)}$

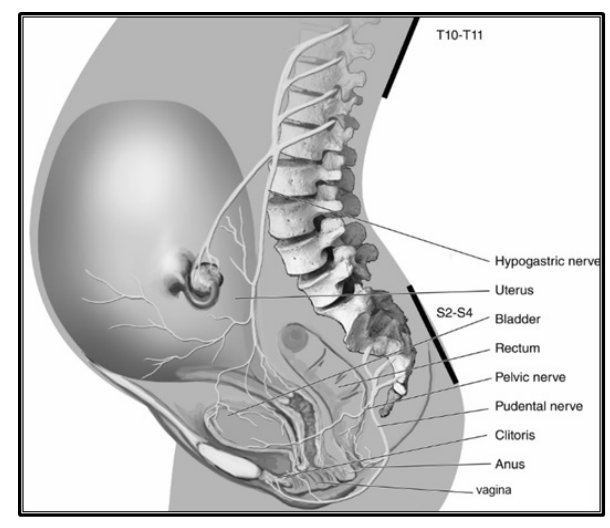

Fig. 2. Pain pathways in a parturient ${ }^{(7)}$

\section{Stages of labor and delivery process}

The labor and delivery process includes three important stages as detailed below: ${ }^{(1)}$

Stage I begins with frequent uterus contractions (frequent uterine contractions with gradual cervix dilation) and ends with full cervix dilation.

Stage II begins with full cervix dilatation and continues until infant delivery.

Stage III begins with infant delivery and continues until complete expulsion of the placenta and membranes.

Components of labor pain

LP is divided into two kinds, somatic and visceral. In Stages I and II of labor, the cervix has a great role in normal labor. During the early Stages I and II of childbirth, the visceral LP is manifested. The cervix is stretched by pressure during uterine contractions; the lower segment of the uterus is thinned out, stimulating excited nociceptive sensory neurons that innervate the endo-cervix and distal segments (T10-L1). ${ }^{(8)}$

Somatic pain occurs at the end of Stage I and during Stage II. It results from sensory nerve endings, which innervate the surface of vagina, perineum, and cervix, and happens due to distension, stretching, ischemia and stretching of vagina, perineum, and pelvic floor. Regular and rhythmic uterine contractions are more forceful during the active stage through the descent of the fetus. With more dilation of the vaginal cervix, the severity of labor pain increases directly with more frequency, duration, and intensity of uterine contractions. ${ }^{(8,9)}$

The LP manifests during Stage I as visceral pain. Visceral pain is transmitted by small unmyelinated ' $C$ ' fibres which travel with sympathetic fibres and pass through the uterine, cervical and hypogastric nerve plexuses into the main sympathetic chain. ${ }^{(8)}$ The nociceptors of the sympathetic chains pass into the white ramus related to spinal nerves of 
T10-L1 and enter through the dorsal nerve root to the dorsal horn cells of the spinal cord. At the level of caudal and rostral extension with dorsal horn cells, some fibres cross over, which leads to poorly localized pain. ${ }^{(8)}$ LP stage I has been shown to be a pain referred to the anterior abdominal wall and to the back (Figure 3) as the anterior abdomen and low back areas are supplied by the same spinal roots that are receiving pain impulses from the uterus. This pain is dull in character. ${ }^{(1,10)}$



Fig. 3. Dermatome levels of the thigh, hips, perineal area, and lower abdomen. ${ }^{(7)}$

Pain during Stage II labor follows a different pathway from labor Stage I. It has been demonstrated to be somatic pain and is diffused by smooth, myelinated, fast conducting A-delta fibers. Diffusion is carried out through the perineal branches of the posterior cutaneous nerve of the thigh and pudendal nerves to S2-4 nerve derivations. From genitofemoral and ilioinguinal nerve roots through cutaneous branches, somatic fibres carry sensory nerve fibers to L1\&2. The somatic pain comes close to labor, is intensive, and definitely localized to the rectum, perineum, and vagina, which radiate to $\mathrm{T} 10$ and $\mathrm{L} 1$ dermatomes with more resistance to opioid medications than visceral pain. ${ }^{(8,11)}$

It is important to realize that these two types of sensation are not mutually exclusive: pain associated with labor Stage I does not stop miraculously with the entry into labor Stage II, but it is often superseded by pain resulting from distention of the perineum due to the descent of the fetus's head. ${ }^{(12)}$

Briefly, all somatic and visceral nerve impulses pass into the spinal cord through dorsal horn cells, which have been handled and transferred to the spinothalamic tract, and travel to the brain in an orderly manner. Transmission of the impulses to limbic and hypothalamic systems determines the autonomic and emotional responses related to pain.

\section{Acknowledgments}

This publication was supported by the Deanship of Scientific Research at Prince Sattam bin Abdulaziz University.

\section{Competing Interests} interests.

The authors declare that they have no competing

\section{References}

1. Datta S, Kodali BS, Segal S. Obstetric Anesthesia Handbook. Fifth Ed. Springer Science+Business Media, LLC, 2010.

2. Hale S, Hill CM, Hermann M, Kinzig A, Lawrence C, McCaughin N, Parker C. Analgesia and Anesthesia in the Intrapartum Period. Nurs Womens Health. 2020 Feb;24(1):e1-e60. doi: 10.1016/j.nwh.2019.12.002.

3. Nguyen V, Tiemann D, Park E, Salehi A. Alpha-2 Agonists. Anesthesiol Clin. 2017 Jun;35(2):233-245. doi: 10.1016/j.anclin.2017.01.009.

4. Wong CA. Advances in labor analgesia. Int J Womens Health. 2010 Aug 9;1:139-54. doi: 10.2147/ijwh.s4553.

5. Omo-Aghoja L. Maternal and fetal Acid-base chemistry: a major determinant of perinatal outcome. Ann Med Health Sci Res. 2014 Jan;4(1):8-17. doi: 10.4103/21419248.126602.

6. Jurna I. Geburtsschmerz-Entstehung, Erregungsleitung und Folgen [Labor pain-causes, pathways and issues.]. Schmerz. 1993 Jun;7(2):79-84. German. doi: 10.1007/ BF02527864. [Article in German]

7. Campling G. Uterine physiology. Anaesth. Intensive Care Med. 2008;9:122-123.

8. Labor S, Maguire S. The Pain of Labour. Rev Pain. 2008 Dec;2(2):15-9. doi: 10.1177/204946370800200205.

9. Origoni M, Leone Roberti Maggiore U, Salvatore S, Candiani M. Neurobiological mechanisms of pelvic pain. Biomed Res Int. 2014;2014:903848. doi: 10.1155/2014/903848.

10. Jetti R, Kadiyala B, Bolla SR. Anatomy, Back, Lumbar Sympathetic Chain. [Updated 2020 Aug 10]. In: StatPearls [Internet]. Treasure Island (FL): StatPearls Publishing; 2021 Jan-. Available from: https://www.ncbi.nlm.nih.gov/books/ NBK539914/

11. Patel AP. Anatomy and physiology of chronic scrotal pain. Transl Androl Urol. 2017 May;6(Suppl 1):S51-S56. doi: 10.21037/tau.2017.05.32.

12. Kovavisarach E. Pain in labor. J Med Assoc Thai. 2004 Oct;87 Suppl 3:S207-11. 